Debatte

2020, Band 8, Heft $1 / 2$

Seiten 167-176

zeitschrift-suburban.de

10.36900/suburban.v8i1/2.564

Lisa Vollmer, Boris Michel

,Wohnen in der Klimakrise.

\title{
Stadtökologie statt Ökologie
}

Kommentar zu Lisa Vollmer und Boris Michel „Wohnen in der Klimakrise.

Die Wohnungsfrage als ökologische Frage“

Die Wohnungsfrage als

ökologische Frage'

Kommentare von:

Andreas Koch, Lukas Häfner,

Anton Brokow-Loga, Miriam

Neßler, Michael Mießner,

Matthias Naumann, Katrin

Großmann, Sören Weißermel,

Rainer Wehrhahn, Klaus

Geiselhart, Bernhard

Hohmann, Thomas Höflehner,

Andreas Koch

Andrea Jany und Marc Amann

\section{Vorbemerkung}

Die nachfolgenden Überlegungen zu einer Debatte der urbanen ,Wohnungsfrage als ökologische Frage' beruhen auf zwei inhaltlichen Positionierungen und gehen räumlich von der kapitalistisch geprägten mitteleuropäischen Stadt aus. Zum einen wird, in Anlehnung an Hartwig Berger (2003), Stadtökologie als politische Ökologie des Urbanen verstanden und damit wesentlich als Gesellschaftsanalyse und Gesellschaftskritik. Eine so verstandene Stadtökologie rückt nicht die biotischen oder technischen Faktoren für nachhaltiges (Zusammen-)Leben ins Zentrum, sondern die sozialen, politischen und kulturellen Grundsätze und Mechanismen, die für die städtischen Lebensformen prägend sind. Bergers vor knapp 20 Jahren geäußerte Kritik scheint auch heute kaum an Relevanz eingebüßt zu haben:

„Dass Stadtökologie direkt Gesellschaftsanalyse und Gesellschaftskritik ist, wird in Öffentlichkeit und Politik nicht begriffen. Die eigentlich triviale Wahrheit, dass nicht, die Umwelt', sondern die mit ihr hantierende Gesellschaft Problem und Patient ist, wird verdrängt.“ (Berger 2003: 31)

Zum anderen wird die Wohnungsfrage als Teil einer kritischen ökologischen Gesellschaftsanalyse nicht mit dem Begriff der ,Krise“ assoziiert, da einer Krise eine inhärente Zeitlichkeit und ein Wendepunkt - zum besseren oder schlechteren - zugrunde liegen. Dies ist im Falle des Wohnens nicht gegeben. Stattdessen sind Wohn- und Wohnungsprobleme in Agglomerationsräumen struktureller Natur, die mit dem Industriekapitalismus ihren Anfang nahmen und sich bis heute, wenngleich in anderer Form und Weise, fortgesetzt haben. Mit der arbeitsteiligen industriellen Massenproduktion setzte eine räumliche Trennung von Arbeiten und Wohnen ein, die bürgerliche Kleinfamilie setzte sich sukzessive als dominanter Haushaltstyp durch, und Privateigentum an Boden und Kapital entschied über Wohnoptionen. All dies brachte neue klassenspezifische Wohnformen hervor, deren grundlegenden Strukturen und Funktionen sich bis heute kaum verändert haben. In Analogie zur 
Einschätzung von Xaver von Cranach (2019: 54): „[e]s gab keine Flüchtlingskrise, es gab Menschen, die flohen und die hier ankamen [...]. Es war eine kausal absolut erklärbare Situation“, lässt sich schließen: es gibt keine Wohn(ungs)krise. Es gibt jedoch ein strukturelles, regional variierendes Wohn(ungs)problem, das sich der kapitalistischen Verwertungslogik verdankt. Beide Positionierungen, die sozialen Verhaltensmuster und die ökonomischen Marktmechanismen, sollen helfen, einige der Schwierigkeiten des urbanen Wohnens in der gegenwärtigen Phase der Klimakrise, die tatsächlich eine Krise ist, zu verstehen.

Von folgenden Kernthesen wird im Beitrag ausgegangen:

- Die Funktionen der Wohnung und des Wohnens haben sich mit der Finanzkrise deutlich in Richtung des Warencharakters verschoben. Die Funktion der sozialen Infrastruktur gerät dadurch weiter unter Druck.

- Die Struktur des Wohnungsmarktes hat sich durch diese Funktionsverschiebung weiter verfestigt. Es dominieren privatwirtschaftliche Interessen des Profits und der Renditeerwartung mit Nebenfolgen der touristischen Kurzzeitvermietung, Zweitwohnungen und des Leerstands.

- Gestützt werden Strukturerhalt und Funktionsverschiebung durch eine am (vermeintlichen) Bedürfnis der Mittelschicht orientierte, dominante Kontinuität der Wohnungsarchitektur am Modell der Kleinfamilie, obwohl der gesellschaftliche Wandel eine Diversifizierung von Wohn(ungs)modellen nahelegen würde.

- Diese politisch-ökologischen Bedingungen haben Konsequenzen für eine ökologische Ausrichtung des Wohnens in der Klimakrise. Denn diese Bedingungen bedeuten, (1) dass mehr vom selben (alten) Modell gebaut wird; (2) dass überhaupt mehr Wohnungsbau als Lösung des Wohnungsproblems gesehen wird; (3) dass ,ökologisches Bauen' gewinnbringend zur Lösung der ökologischen Probleme stilisiert wird. Stattdessen müssten als Ziele angedacht werden: (a) auf regionaler Ebene ein Wohnungsbaumoratorium; (b) ebenfalls auf regionaler Ebene eine rechtsverbindliche Regionalplanung des Wohnungsbaus; (c) auf lokaler Ebene eine Diversifizierung des Wohnens in Richtung kollektiver Wohnformen.

\section{Wohnungsfunktionen}

Nach Jürgen Hasse (2019: 13) stellen sich für das 21. Jahrhundert folgende Fragen als vorrangig heraus: „Was tut man, wenn man wohnt? Wer wohnt mit wem zusammen? Wie wird Wohnen erlebt? Wie kommt man zur Wohnung?“. Fragen des nachbarschaftlichen Zusammenlebens, des Zugangs und der Repräsentation des Wohnens sind zudem um jene nach der Räumlichkeit (wo wohnt man?) und Zeitlichkeit (wie lange wohnt man wo?) zu ergänzen. Sie sind jedoch vor allem mit den Funktionen der Wohnung in einen $\mathrm{Zu}-$ sammenhang zu bringen, die sich, nicht zuletzt durch die Finanzkrise 2008, deutlich in Richtung der Wohnung als handelbare Ware verschoben haben. Wohnungsmärkte unterliegen insofern primär kapitalistischen Verwertungslogiken, mit dem Ziel der profitorientierten Kapitalakkumulation (Harvey 2013: 88 ff.) oder der wirtschaftlichen Absicherung im Alter, wie 
Susanne Heeg (2013) dies unter dem Gesichtspunkt der Responsibilisierung, das heißt der individualisierten Verantwortungsübertragung der eigenen Vorsorge für die Zeit nach der Erwerbsarbeit, diskutiert. Daraus resultieren eine Zunahme von Zweitwohnsitzen, Leerstand oder der temporären touristischen Nutzung. Die Funktion der Wohnung als Teil der sozialen Infrastruktur gerät damit zunehmend unter Druck.

Die Ursprünge dieser Entwicklung liegen historisch in der Phase der Industrialisierung, die durch wachsende Arbeitsteilung und Funktionstrennung charakterisiert ist. Trotz aller Reformbestrebungen im Kontext der sozialen und der Wohnungsfrage, die sich frühzeitig mit diesen Umwälzungen etablierten, spiegeln sich bestimmte soziale Leitbilder auf den Wohnungsmärkten bis heute wider. Zu diesen Leitbildern gehören neben anderen Charakteristika das Wohnen als Kleinfamilie, die Wohneigentumsbildung und das Einfamilienhaus im suburbanen Raum (Beck/Reutlinger 2019: $97 \mathrm{ff}$.). Sie sind sichtbarer Ausdruck der hier diskutierten These, dass Wohnungsprobleme strukturelle Probleme sind, die für eine ökologische Ausrichtung der Wohn- und Wohnungsfrage mitzudenken sind. Der angesprochene Wandel der Funktionen der Wohnung wie des Wohnens trägt eher zur Verfestigung als zur Veränderung gewachsener Strukturen bei. Mit ,gewachsenen Strukturen ' ist dabei gemeint, dass erstens Marktmechanismen als geeignetes Aushandlungsmodell zur Bedürfnisbefriedigung nach Wohnraum erachtet werden, dass zweitens auch der öffentlich geförderte Wohnbau gezwungen ist, eine am Privateigentum orientierte Wohn(ungs)- und Bodenpolitik zu verfolgen und damit lediglich in der Lage ist, Symptombekämpfung zu betreiben. Drittens folgt die Wohnungsarchitektur weiterhin dem traditionellen Ideal der Zweigenerationenfamilie und begünstigt damit ebenso den handelbaren Warencharakter der Wohnung. Ausgangspunkt der nachfolgenden Ausführungen bildet der zuletzt genannte Punkt (Kleinfamilie als vorherrschendes Leitbild), mit dem dann auf ausgewählte eigentumstheoretische Punkte eingegangen wird. Gerade das Leitbild der Kleinfamilie versinnbildlicht den Warencharakter der Wohnung, weil es bestrebt ist, kleine Einheiten mit klarer eigentumsrechtlicher Zuschreibung und individualisiertem Tauschwert zu favorisieren. Damit folgt es dem Prinzip der Kleinteiligkeit und Unteilbarkeit, wie wir es vom Auto, der Bohrmaschine oder der Gartennutzung kennen. Wohnungen, die kollektive Wohnformen wie beispielsweise social cohousing ermöglichen, würden sich aufgrund ihrer komplizierteren eigentumsrechtlichen Zuschreibung nicht so leicht kapitalisieren lassen.

\section{Dynamischer sozialer Wandel...}

Die starre Ausrichtung des Wohnungsbaus an diesen sozialen Leitbildern fokussiert damit reduktionistisch die (vermeintlichen) Bedürfnisse der Mittelschicht. Die Unterschicht wird, wie Sylvia Beck und Christian Reutlinger (2019) für die Soziale Arbeit mit ihrer ,Normalisierungsfunktion“ herausstellen, über Unterstützungs- und Steuerungsnormen zu integrieren versucht. Während Unterstützung auf sozial- und rechtsstaatliche Einbindung (z. B. Wohnbeihilfen) abzielt, erfolgt die regulierende Steuerung über Erwerbsarbeit und Eigentum und damit über den klassischen, sozialen Kompromiss 
des Industriekapitalismus' (Castel 2011, zit. nach Beck/Reutlinger 2019: 118). Beide Schichten haben sich dabei, wenngleich qualitativ anders als ihre industriekapitalistischen Vorgängerinnen, zu neuen Klassen gewandelt. Die Polarisierung zwischen neuer akademisch-kultureller Mittelklasse und neuer Unterklasse, so Andreas Reckwitz (2017: 280 f.), betrifft auch die Wohnbedürfnisse und Wohnansprüche.

Mit der neuerlichen Metamorphose von Schichten zu Klassen haben sich, sowohl innerhalb als auch zwischen ihnen, deren Lebensstile weiter ausdifferenziert. Im Ergebnis führt dies zu einem komplexen Konglomerat unterschiedlicher und heterogener Lebens- und Wohnformen. Für die akademische Mittelkasse charakteristisch sind die erweiterten Möglichkeiten sozialer und räumlicher Mobilität oder die weiter zunehmende Auslagerung haushaltsorientierter Dienstleistungen (wie Essenszubereitung, Kleiderreinigung, Kinder- und Tierbetreuung oder Einkaufen), da viel Arbeits-, Regenerationsund Kulturzeit außerhalb der eigenen Wohnung verbracht wird. Zugleich werden aber auch viele Tätigkeiten des Arbeitens, Lernens, Versorgens oder Ausruhens (wieder) in die eigene Wohnung integriert. Wohnen folgt daher nicht einem eindimensionalen, raumzeitlich linearen Funktionsverständnis und ist insofern räumlich wie funktional fluider geworden. Ein Indikator ist das Wachstum der haushaltsorientierten Service Economy, die, wie angedeutet, vielfältige Möglichkeiten der Auslagerung und Rückverlagerung offeriert (Bartmann 2016). Die Fluidität des Wohnens zeigt sich exemplarisch aber auch an den multilokalen Wohnformen, temporären Wohnsitzen oder auch den lokalen Umzugsdynamiken in Abhängigkeit biographischer Veränderungen, die ebenfalls zugenommen haben. Beispielsweise variieren die Umzugsquoten in den 20 größten Städten Deutschlands im Jahr 2017 zwischen 5,9 Prozent in Berlin und 10,6 Prozent in Münster und Nürnberg (vgl. Statista 2020). Bei einer durchschnittlichen Umzugsdynamik von 8,5 Prozent zeigt sich, dass gerade in den teuren Städten die residentielle Mobilität vergleichsweise gering und im Vergleich der letzten Jahre weiter gesunken ist.Zunehmende Sesshaftigkeit mangels Alternativen unterminiert dabei nicht nur lebensstilbezogene, sondern ebenso biographische Anpassungsmöglichkeiten der Wohnbedürfnisse, die insbesondere auch eine Reduktion der Wohnfläche im Alter inkludiert.

Für jene Menschen, deren Einkommen niedrig(er) oder deren Mobilität aus unterschiedlichen Gründen eingeschränkt(er) ist (nach Reckwitz die neue Unterklasse), hat sich ebenfalls eine Pluralisierung der Lebens-, Arbeitsund Wohnformen herausgebildet. Zum einen lässt sich ein Bemühen um Wohnstandortfixierung im urbanen Raum dort erkennen, wo es für sie noch leistbar ist, da städtische Arbeitsmärkte gegenüber ländlichen ein auch für gering(er) Qualifizierte stärker diversifiziertes Angebot an Arbeitsplätzen bereitstellen. Sofern es möglich bleibt in der Stadt zu wohnen, wird ökonomisch erzwungene Sesshaftigkeit weniger als Problem denn als Chance der Absicherung erreichter Lebensqualität gesehen - ein auch unter dem Gesichtspunkt des Erhalts sozialer Beziehungen nicht zu vernachlässigender Grund. Angesichts hoher und steigender Preise auch im öffentlich geförderten Wohnbau, stößt dieses Bemühen jedoch immer häufiger an seine Grenzen. In der österreichischen Stadt Salzburg zum Beispiel liegt derzeit der Quadratmeterpreis für gemeinnützige Mietwohnungen bei 10,86 Euro, für 
private bei 15,60 Euro (Koch 2020). Da auch andere wohnpolitische Maßnahmen wie Mietobergrenzen oder Mietpreisbremsen nicht die erwünschte Wirkung brachten, bleibt als Alternative dann nur das (derzeit noch) günstigere weitere Stadtumland, verbunden mit höheren Fahrtkosten und -zeiten sowie Emissionen an Abgasen und Lärm. Zum anderen erzwingen hohe Wohnungspreise unfreiwillige mobile Wohnformen wie transregionale und transnationale Migration oder temporäre Wohnaufenthalte an Orten mit geringer(er) Lebensqualität.

\section{4. ...und träger Wandel der Wohn- und Wohnungsformen}

Eine stadtökologische Debatte über ,Wohnen in der Klimakrise' müsste nun an diesen wohnsozialen und wohnungswirtschaftlichen Entwicklungen kritisch anschließen, was nachfolgend mit einigen wenigen Gedanken versucht wird.

Das gegenwärtige Wohnleitbild hält weiterhin an der bürgerlichen Kleinfamilie als gesellschaftlichem Ideal fest. Diese Feststellung trifft selbst für steigende Zahlen von Singlehaushalten junger und älterer Menschen wie für Alleinerzieher_innen-Haushalte zu, die lediglich in reduzierter Form das wohnsoziale Idealbild repräsentieren. Und sie wird auch nicht durch eine wachsende Zahl von Gegenbeispielen widerlegt, die es zweifelsohne in Form von Mehrgenerationenhäusern, verschiedenartigen Wohnungs-, Bauund Umbaugemeinschaften sowie digitalen Wohnplattformen oder Wohnungstauschbörsen gibt (Fuhrhop 2015: 125 ff.). Denn gerade weil sich die Umsetzung derartiger Initiativen wohn(ungs)politisch so schwierig gestaltet, kann dies als Indiz eines trägen Wandels der Wohn- und Wohnungsformen gewertet werden und ist somit Ausdruck einer Unterbewertung des Wohnens in sozialräumlicher Hinsicht.

Anstatt dem Credo eines „Bauen, Bauen, Bauen“ zu folgen, wäre es, wie eingangs formuliert, stadtökologisch klüger, den Möglichkeitsspielraum um die eben genannten Wohnformen noch zu erweitern und eine stärkere Mischung von mobilen und immobilen Wohnformen, unter anderem in Abhängigkeit der biographischen, familiären oder arbeitsbedingten Situationen, anzustreben. Beispiele wären ein Angebot an öffentlichen Zweitwohnsitzen, an Co-Working angelehntes Co-Living oder Wohnplattformen für an wechselnden Orten arbeitende Menschen. Derartige Projekte können durch flankierende Maßnahmen wie Wohnkoordination oder soziale Startbegleitung, wie sie es für neue Wohnsiedlungen bereits gibt, zu einer Förderung und positiven Bewertung des nachbarschaftlichen Zusammenlebens beitragen. Auch wenn Urbanität auf diese Weise ein Stück weit inszeniert wird (Smigiel/Koch 2018), so rufen solche Maßnahmen zugleich den Gedanken des Wohnens als sozialer Infrastruktur wieder stärker in Erinnerung.

\section{Soziale und kulturelle Überbewertung des Wohnens}

Stattdessen lässt sich neben der wohnungsarchitektonischen Unterbewertung auch eine gesellschaftspolitische Überbewertung traditioneller Wohnmodelle beobachten. Ein Indiz hierfür findet sich in der ausgeprägten Betonung der 
Individualisierung von Wohnbedürfnissen. So negieren Beck und Reutlinger (2019: 42) zwar nicht die Notwendigkeit der Gestaltung des eigenen Lebens und Wohnens, betonen jedoch stärker die Gestaltungsfreiheiten und identitätsstiftenden Potenziale spätmodernen Wohnens: „So gedacht erklärt sich spätmodernes Wohnen (nur noch) aus der individuellen Lebenssituation und daran geknüpften Vorstellungen, Bedarfe und Bedürfnisse [...]“. Diese Form der Inszenierung ist jedoch trügerisch und politisch-ökologisch problematisch. Denn die sich potenzierenden Möglichkeiten an Lebensweisen und -stilen führen angesichts empfundener Überforderungen eher zu Versuchen der Nachahmung und Standardisierung (,Ikeaisierung' des Wohnens). Um dem aufoktroyierten Bedürfnis nach Individualität im Wohnen dennoch nachzukommen, werden Einrichtungsgegenstände in kurzen Zyklen ausgetauscht, mehr von ihnen gekauft oder mehr repräsentativer Platz beansprucht. Alles das sind Ausdrucksformen, die dem ,[...] fatalen Vorbild der klassischen Moderne [des Industriekapitalismus; A.K.] folgen [...]“ (Fuhrhop 2015: 107) und dem Wohnen in der Klimakrise zuwiderlaufen.

Hinzu kommt, dass die suggerierte Individualität von Lebens- und Wohnstilen einer mittlerweile typischen Verengung der Perspektive auf die Mittelschicht entspricht. Individualität muss man sich leisten können und wollen. Einkommensschwächere Haushalte können dies in der Regel nicht, da sie einen wachsenden Anteil ihres Einkommens für Miete oder Kreditrate aufbringen müssen. Um im Streben nach Anerkennung und Identitätsartikulation nicht exkludiert zu werden, versuchen auch diese Haushalte, Teil des Steigerungsspiels um ein Mehr an Gegenständen und Wohnfläche zu bleiben.

Nahrung erhält die inszenierte Individualität durch eine entsprechende mediale Inszenierung des Wohnens. Die Darstellungen von Wohnungen in einschlägigen Werbeprospekten lädt das Grundbedürfnis Wohnen zu einer hochstilisierten Exklusivität und einer Sphäre der Selbstverwirklichung auf, die mit dem Bild des freistehenden Hauses im Grünen und umgeben von endloser Natur helfen soll, Wohnen als familiäres Idyll zu vermarkten. Selbst ökologisches Bauen und Einrichten bedient sich dieser Strategie eines als legitim erachteten Konsums ohne Reue, wie Daniel Fuhrhop (2015: 32) mit Verweis auf einen Architekten hervorhebt: „Nachhaltiges Bauen darf nichts mit Entsagung zu tun haben“.

\section{Wohnen als sozialer Infrastruktur Nachdruck verleihen}

Die sozialkulturelle Überbewertung der kleinfamiliären Wohnformen erscheint angesichts der gesellschaftlichen Veränderungen unzeitgemäß, aus der Sicht der kapitalistischen Akkumulationslogik jedoch nur konsequent. Eine stadtökologische Kritik sollte sich nachdrücklich dafür einsetzen, dem Wohnen als soziale Infrastruktur wieder seinen zentralen Stellenwert zuzuweisen, so sehr auch das Erfordernis der Wohnung als Vorsorgeobjekt mangels alternativer renditeträchtiger Kapitalanlagen aus individueller Perspektive verständlich erscheint. Denn, wie Andrej Holm (2019: 107) treffend hervorhebt: „Für eine soziale Wohnungsversorgung gibt es [...] innerhalb der Marktlogik keinen Anreiz. [...] Auf Märkten zählen weder Bedürfnisse noch Bedarf, sondern allein die Nachfrage“. 
Hierfür gibt es bereits heute eine Vielzahl an Umsetzungsmöglichkeiten wie gemeinnütziger, kommunaler oder genossenschaftlicher Wohnungsbesitz, Bekämpfung von Wohnungsleerstand oder zweckwidrig genutzten Wohnräumen, Reduktion von Zweit-, Neben- oder touristisch vermarkteten Wohnungen, Umwidmung von leerstehenden oder unterausgelasteten Büround Gewerbegebäuden. Ein gemeinsamer Nenner all dieser Maßnahmen liegt darin, den als politisches Heilmittel gepriesenen Wohnungsneubau zu überdenken. Fuhrhop (2015) liefert hierfür eine Vielzahl an Argumenten, die es wert sind, in Debatten zur, Wohnungsfrage in der Klimakrise aufgegriffen zu werden. Angesichts des anhaltend hohen Flächenverbrauchs, insbesondere in städtischen Agglomerationsräumen, würde bereits ein temporäres Wohnbaumoratorium helfen, den Lebensraum heutiger und künftiger Generationen mit der gebotenen Nutzungssensibilität anzuerkennen.

Neben ökologiepolitischen Strategien, die auf eine Dekommodifizierung der Wohnung abzielen, sind Überlegungen zu einer Bodenreform und einer Reform der in kommunaler Hand liegenden Bauplanung anzustellen.

„Die Frage des Bodeneigentums muss daher wieder Thema ökologischer Stadtentwicklung werden. Aus Gründen der ökologischen Nachhaltigkeit ist ein Bodenrecht kontraproduktiv, das diese wichtige und nicht erneuerbare Naturressource zu einer handelbaren Ware und zum Gegenstand privatrechtlichen Gewinns macht.“ (Berger 2003: 66)

Die Verfügungsgewalt über Grund und Boden in öffentliche oder gesellschaftliche Hände zu legen, löst für sich genommen zwar noch nicht die Forderung einer nachhaltigen Bodenpolitik ein. Dafür sind in einer kapitalistisch ausgerichteten Wirtschafts- und Gesellschaftsverfassung umfassendere und grundsätzliche politische Veränderungen notwendig. Dies zeigt sich, mit Blick auf Österreich, exemplarisch daran, dass viele Gemeinden in der Vergangenheit Bauland ausgewiesen, den rechtlichen Folgen dieser Umwidmung jedoch nicht Nachdruck verliehen haben - nämlich zu bauen. Damit wurde der Bodenspekulation mit Hilfe der öffentlichen Hand Vorschub geleistet. Eine Kommunalisierung von Grund und Boden würde jedoch den Handlungsspielraum von Wohnkollektiven und politisch legitimierten Institutionen erhöhen, einen Ausgleich zwischen privat- und gemeinwirtschaftlichen und zwischen wirtschaftlichen und gesellschaftlichen Interessen zu erzielen. Hierdurch ließen sich dann auch stadtökologische Maßnahmen wie Funktionsmischung (Stadt der kurzen Wege, Wohnung als multifunktionaler Ort), sozialräumliche Mischung (nachbarschaftliche Konfrontation unterschiedlicher sozialer Lagen, Urbanisierungspolitik) oder umweltverträgliche Mobilitätsformen politisch priorisieren.

Um die Bodenreform ihrem Ziel näher zu bringen, sind ergänzende Maßnahmen einer Reorganisation der Stadtplanung und Stadtentwicklung unumgänglich. Solange die Bauleitplanung im kommunalen Hoheitsrecht verankert bleibt, wird jede Stadt versucht und gezwungen sein, ihre eigenen lokalen Interessen über die überörtlichen und übergeordneten zu stellen. Mit einer subsidiären Verlagerung der planungsrechtlichen Kompetenzen auf eine regionale Ebene müssten zugleich die Einnahmen der Städte auf eine andere Grundlage gestellt werden, was angesichts dann auch zu erwartender Ausgabensenkungen durch abnehmende wettbewerbsbedingte 
Entscheidungen (für Bau und Erhalt von Infrastrukturen, Personal und Geräten) und regionaler Synergien im Bereich des Möglichen liegt. Mit einer verbindlichen Regionalplanung in den Bereichen Baulandausweisung und Wohnbau (wie auch gewerblicher Bau) wären Gemeindefusionen keine zwingende Alternative, sodass die lokale demokratische Legitimation nicht verloren ginge, vielmehr ein Interessenausgleich über ebenjene lokalen politischen Institutionen zu erzielen wäre. Mit einer regionalen planungsrechtlichen Verbindlichkeit ließe sich zudem die Beschränkung auf territoriale Raumeinheiten aufbrechen, indem räumliche Einheiten problemzentriert flexibel gebildet würden, zum Beispiel nach funktionalen Gesichtspunkten für bestimmte Infrastrukturen (Hoffmann-Axthelm 2016: 61 ff.).

\section{Resümee}

Die manifeste Klimakrise offenbart die strukturellen Probleme des Wohnens aufs Neue. Die Kommodifizierung der Wohnung als Vorsorge- und/oder Renditeobjekt hat im letzten Jahrzehnt weiter zugenommen, worunter ihre Eigenschaft, Teil der sozialen Infrastruktur zu sein, weiter gelitten hat. Mit der Klimakrise werden auch die mit der Wohn- und Wohnungsfrage in $\mathrm{Zu}-$ sammenhang stehenden Aspekte neuerlich virulent. Die Ausrichtung der Wohnung an der Kleinfamilie, die Propagierung von Wohneigentum oder die Inszenierung des Wohnens als Mittel individualistischer Selbstdarstellung laufen einer adäquaten Antwort auf die Klimakrise zuwider. Vielmehr tragen sie zu einem weiterhin steigenden Ressourcen- und Flächenverbrauch bei. Kleine Wohnungen sind nicht nur unter Kapitalisierungsgesichtspunkten besonders beliebt, sie benötigen auch die relativ zur Personenzahl gerechnet meiste Fläche. Jede dieser kleinfamiliär ausgerichteten Wohnungen braucht eine eigene Küche und Haushaltsgeräte zur exklusiven Nutzung. Diese Geräte sind somit unterausgelastet und unterliegen dennoch einer hohen Zyklizität der Wiederanschaffung (Welzer 2013).

Nachhaltig wäre vielmehr eine „Ethik vorsorgender Selbstbeschränkung“ (Berger 2003: 14), anstatt mehrWohnungen zu bauen. Mit dieser Einschätzung soll nicht die Verantwortung für ökologisches Bauen ausschließlich auf jede/n Einzelne/n delegiert werden. Vielmehr sollten alle Beteiligten prüfen, ob mit dem Festhalten an tradierten sozialen Leitbildern die passende Antwort auf die aktuellen Funktionsverschiebungen des Wohnens und der Wohnung und die Folgen des Klimawandels gegeben wird.

\section{Autor_innen}

Andreas Koch ist Sozialgeograph und beschäftigt sich mit Fragen der Armut und sozialen Ungleichheit in urbanen sowie dünnbesiedelten Räumen Europas, Kanadas und Australiens. andreas.koch@sbg.ac.at 


\section{Literatur}

Bartmann, Christoph (2016): Die Rückkehr der Diener. Das neue Bürgertum und sein Personal. München: Hanser.

Beck, Sylvia / Reutlinger, Christian (2019): Die Wiederkehr der Wohnungsfrage. Zürich: Seismo.

Berger, Hartwig (2003): Entgrenzte Städte. Zur politischen Ökologie des Urbanen. Münster: Westfälisches Dampfboot.

Castel, Robert (2011). Die Krise der Arbeit. Neue Unsicherheiten und die Zukunft des Individuums. Hamburg: Hamburger Edition HIS Verlags-GmbH.

Fuhrhop, Daniel (2015): Verbietet das Bauen! Eine Streitschrift. München: Oekom.

Harvey, David (2013): Rebellische Städte. Berlin: Suhrkamp.

Hasse, Jürgen (2019): Was bedeutet es zu wohnen? In: Bundeszentrale für politische Bildung (Hg.): Gesucht! Gefunden? Alte und neue Wohnungsfragen. Bonn: bpb, 12-21.

Heeg, Susanne (2013): Wohnungen als Finanzanlage. Auswirkungen von Responsibilisierung und Finanzialisierung im Bereich des Wohnens. In: sub\urban. zeitschrift für kritische stadtforschung, 1/1, 75-99.

Hoffmann-Axthelm, Dieter (2016): Lokaldemokratie und Europäisches Haus. Bielefeld: Transcript.

Holm, Andrej (2019): Wiederkehr der Wohnungsfrage. In: Bundeszentrale für politische Bildung (Hg.): Gesucht! Gefunden? Alte und neue Wohnungsfragen. Bonn: bpb, 98-111.

Koch, Andreas (2020): Wohnen in der Stadt Salzburg. Zum Verhältnis der Wohnung als Ware und dem Wohnen als soziale Infrastruktur. In: Salzburger Jahrbuch für Politik 2020 (im Druck). Wien: Böhlau.

Reckwitz, Andreas (2017): Die Gesellschaft der Singularitäten. Berlin: Suhrkamp.

Smigiel, Christian / Koch Andreas (2018): Formen von Urbanität in der kapitalistischen Moderne und ihre Effekte. In: polylog 39, 19-33.

Statista (2020): Umzugsquoten in den größten Städten Deutschlands im Jahr 2017. https://de.statista.com/statistik/daten/studie/731899/umfrage/umzugsquote-in-dengroessten-staedten-deutschlands/ (letzter Zugriff am 12.1.2020)

Vollmer, Lisa / Michel, Boris (2020): Wohnen in der Klimakrise. Die Wohnungsfrage als ökologische Frage: Aufruf zur Debatte. In: sub \urban. zeitschrift für kritische stadtforschung, 8/1-2, 163-166.

Von Cranach, Xaver (2019): Welche Krise? In: Die Zeit, 27.12.2019, 54.

Welzer, Harald (2013): Selbst denken. Eine Anleitung zum Widerstand. Frankfurt am Main: Fischer. 